\title{
Stereospecific Epitaxial Growth of Bilayered Porous Molecular Networks
}

Yuan Fang, ${ }^{a, f}$ Benjamin D. Lindner, ${ }^{b}$ Iris Destoop, ${ }^{a}$ Takashi Tsuji, ${ }^{b}$ Zhenzhe Zhang, ${ }^{f}$ Rustam Z.

Khaliullin, ${ }^{f}$ Dmitrii F. Perepichka, ${ }^{f}$ Kazukuni Tahara, ${ }^{b, c}$ Steven De Feyter, ${ }^{a *}$ and Yoshito Tobe $e^{b, d, e *}$

${ }^{a}$ Department of Chemistry, Division of Molecular Imaging and Photonics, KU Leuven, Celestijnenlaan 200F, Leuven, B-3001, Belgium

${ }^{b}$ Division of Frontier Materials Science, Graduate School of Engineering Science, Osaka University, 1-3 Machikaneyama, Toyonaka, Osaka, 560-8531, Japan

${ }^{c}$ Department of Applied Chemistry, School of Science and Technology, Meiji University, 1-11 Higashimita, Tama-ku, Kawasaki, 214-8571, Japan

${ }^{d}$ The Institute of Scientific and Industrial Research, Osaka University, 8-1 Mihogaoka, Ibaraki, Osaka, 567-0047, Japan

${ }^{e}$ Department of Applied Chemistry, National Chiao Tung University, 1001 Ta Hsueh Road, Hsinchu, 30010, Taiwan

fDepartment of Chemistry, McGill University, 801 Sherbrooke Street West, Montreal, Québec, H3A 0B8, Canada

\begin{abstract}
Stereo-controlled multilayer growth of supramolecular porous networks at the interface between graphite and a solution was investigated. For this study, we designed a chiral dehydrobenzo[12]annulene (DBA) building block bearing alkoxy chains substituted at the 2position with hydroxy groups, which enable van der Waals stabilization in a layer and potential hydrogen bonding interactions between the layers. Bias voltage-dependent scanning tunneling microscopy (STM) experiments revealed the diastereoselectivity of the bilayer with respect to both the intrinsic chirality of the building blocks and the supramolecular chirality of the selfassembled networks. Top and bottom layers within the same crystalline domain were composed of the same enantiomers but displayed opposite supramolecular chiralities.
\end{abstract}




\section{INTRODUCTION}

Self-assembled molecular networks (SAMNs) may spontaneously form on surfaces. In contrast to self-assembled monolayers (SAMs) of alkanethiols chemisorbed on gold as an example, the organization of the molecules in SAMNs only involves noncovalent interactions. The molecules are physisorbed on the surface, very often lying flat on the surface and giving rise to single-crystalline domains of a few atoms thick that can extend over several square micrometers. ${ }^{1}$ Control over the structure and functionality of SAMNs on surfaces or at liquid/solid interfaces has attracted broad interest in the context of nanoscience and nanotechnology. ${ }^{2-3}$ Among the various types of two-dimensional (2D) architectures constructed using molecular self-assembly, nanoporous networks are challenging structures because of the necessity to surmount the inherent tendency of molecules to avoid the formation of porous structures and assemble in a densely packed geometry. However, they are highly attractive for applications in surface templating, nanopatterning, heterogeneous catalysis and separations. ${ }^{4-6}$ Another challenge in this field is the controlled epitaxial growth of SAMNs orthogonal to the substrate, a process with strong relevance to anchoring three-dimensional (3D) metal-organic materials on surfaces (SURMOFs), ${ }^{7-9}$ electronics of semiconducting molecules ${ }^{10}$ and crystal engineering. ${ }^{11}$ In particular, stereochemical information about the first few molecular layers can provide insight into the evolution of crystallization from 2D to 3D systems. ${ }^{12}$

Among the various techniques used to probe the structure of ultrathin films, scanning tunneling microscopy (STM) takes a unique position, as it provides submolecular resolution, even at the interface between a solid substrate and a solution. Bilayers and multilayers were studied using STM for planar $\pi$-conjugated molecules that self-assemble via $\pi$ - $\pi$ interactions ${ }^{13-}$ 22 and for highly polarized molecules undergoing dipole-dipole interactions. ${ }^{22-23}$ Typically, the self-assembled second layer does not have a specific registry to the bottom layer. Specific pointto-point interlayer interactions are desirable to precisely control the interlayer geometry. 
A few studies on the stereochemical relationship in both lateral (parallel to the surface) and longitudinal directions (along the surface normal) of densely packed double layers grown through van der Waals interactions have been performed using chiral molecules with a twisted or helical backbone, such as rubrene, ${ }^{24}[5]$ helicene, ${ }^{25}$ and [7]helicene, ${ }^{26-27}$ under ultrahigh vacuum (UHV) conditions. Because of their nonplanar structures, these molecules are packed via van der Waals contacts in a point-to-point manner in both the lateral and longitudinal directions. They form either a racemate or conglomerate double layer. ${ }^{28}$ More specifically, the overlying second layer has opposite or the same handedness with respect to the intrinsic chirality of the molecules themselves and supramolecular chirality of molecular self-assembly. One study reports the nonlinear chiral amplification effect on bilayers of near-racemic mixtures involving the biased adsorption and organization of the major enantiomer at the liquid/solid interface. $^{29}$

For multilayered porous networks, although a few examples constructed through longitudinal van der Waals or dipole interactions of intrinsically porous macrocyclic molecules have been reported, ${ }^{30-35}$ the geometry of their nonbottom layers is not strictly controlled or not determined. To the best of our knowledge, only two reports have described the formation of porous double layer SAMNs using small nonmacrocyclic molecules, but they do not address chirality. ${ }^{36-37}$

Here, we propose an approach to construct self-assembled porous multilayer films with precise control over the interlayer geometry. For this purpose, we selected alkoxy-substituted dehydrobenzo[12]annulene (DBA) derivatives because of their demonstrated ability to form chiral porous honeycomb patterns via the interdigitation of alkoxy chains (Scheme 1$)^{38-39}$ and tunability of pore size by varying the alkoxy chain length. ${ }^{40}$ We reported the formation of supramolecular networks of a well-defined handedness using chiral DBA (cDBA) molecules, such as CDBA-OC12(S) and CDBA-OC12(R) (Chart 1), with methyl-substituted stereogenic centers, and their roles in inducing and inversing supramolecular chirality of networks of achiral 
DBA molecules. ${ }^{41-43}$ Based on these results, we hypothesized that replacing the methyl groups with hydrogen bonding groups would also lead to the formation of similar porous chiral networks, and furthermore would enforce the formation of porous multilayers (Scheme 1), even in a stereo-controlled manner.

a)

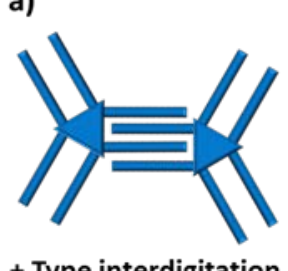

+ Type interdigitation
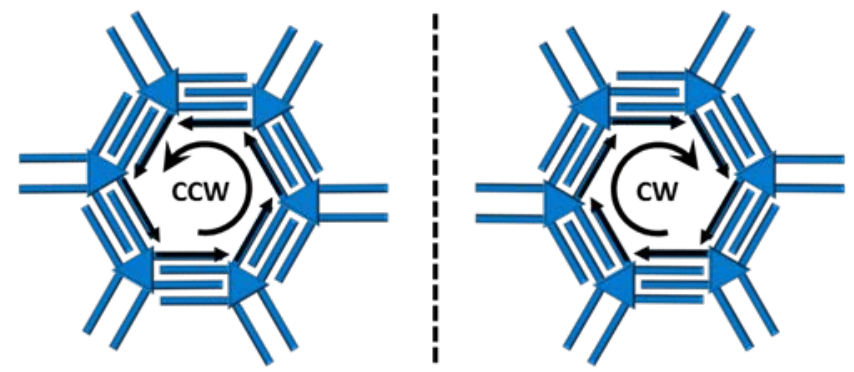



- Type interdigitation

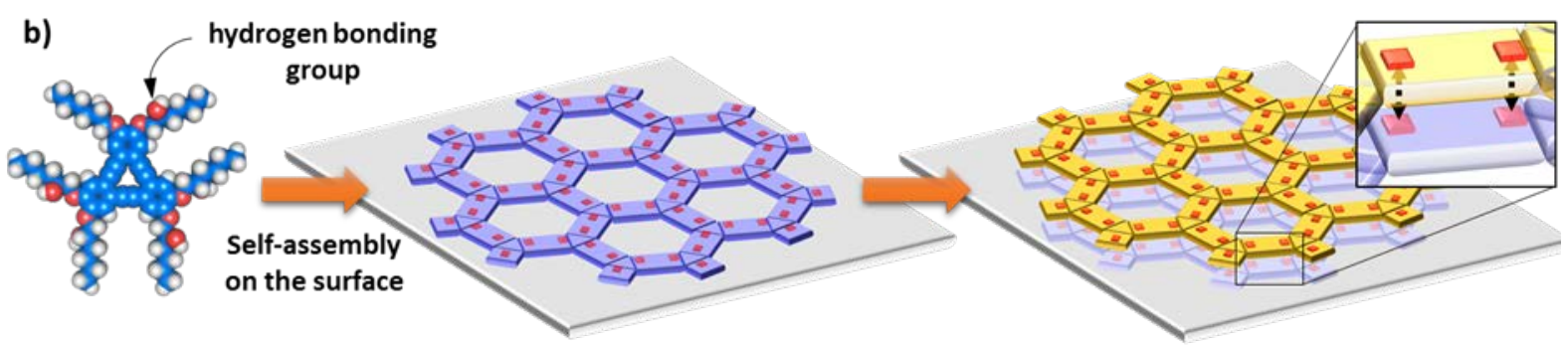

Scheme 1. a) Typical supramolecular pore formed by alkylated DBA molecules. The type of alkyl chain interdigitation determines the handedness of the supramolecular pores and the supramolecular networks. b) Schematic of a self-assembled porous double layer structure; chirality is ignored for simplicity. In the first step, the DBA molecules bearing polar hydroxy groups (red squares) self-assemble into a honeycomb network on the surface via van der Waals interactions between the alkoxy chains. The growth of the second layer is directed by the van der Waals interactions between the two layers, possibly with the involvement of longitudinal hydrogen bonding of the hydroxy groups.

Therefore, we designed the chiral DBA derivatives cDBA-OC6-OH(S) and cDBA-OC6$\mathbf{O H}(\boldsymbol{R})$ with a hydroxy group at the 2-position of each hexyloxy chain (Chart 1). We selected 
hexyloxy chains to promote the formation of the porous supramolecular structure with relatively small pores of approximately $1.7 \mathrm{~nm}$ in diameter, as revealed previously for DBAOC6 (Scheme 1). ${ }^{44}$ Upon adsorption and self-assembly on the surface, the homochiral cDBAOC6-OH(S) and cDBA-OC6-OH(R) compounds are expected to form homochiral porous patterns in either the clockwise (CW) or counter clockwise (CCW) direction through (-) and $(+)$ interdigitation modes of the alkoxy groups, respectively (Scheme 1 and Figure 1), similar to the chiral DBAs with stereogenic methyl groups. ${ }^{41}$ More importantly, the hydroxy substituents of cDBA-OC6-OHs should orient away from or towards the substrate in an alternating manner, thereby promoting the formation of hydrogen bonds and a multilayer structure. Therefore, the growth of the overlying layers may occur in a stereospecific manner with respect to the first layer. The stereoselectivity at each step of self-assembly is summarized in Scheme 2 to illustrate the stereochemical outcomes of the formation of the anticipated multilayer structure.

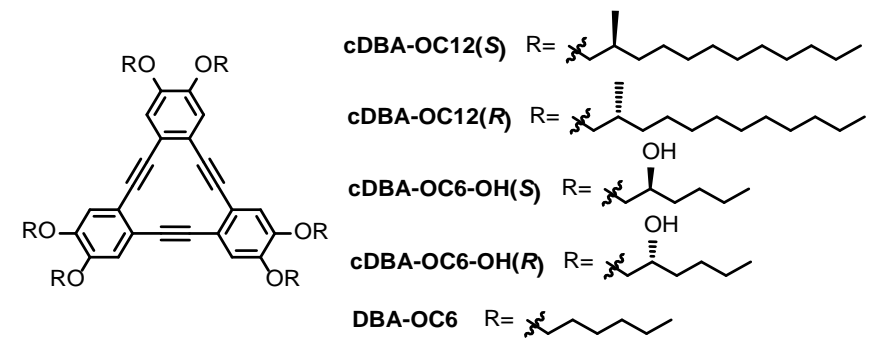

Chart 1. Chemical structures of cDBA-OC12(S), CDBA-OC12(R), CDBA-OC6-OH(S), CDBA-OC6-OH(R), and DBA-OC6.

Indeed, we revealed the formation of a bilayer of cDBA-OC6-OH(S) and cDBA-OC6$\mathbf{O H}(\boldsymbol{R})$ at the interface between graphite and a liquid using bias voltage-dependent STM observations. The enantiomorphism of the bottom layer was identical to chiral DBAs bearing methyl groups on the chiral center [(Steps (1) and (2) in Scheme 2]. Most interestingly, the multilayer growth occurred in a diastereospecific manner between the top and bottom layers 
with respect to both the intrinsic chirality of chiral DBAs, i.e., $S$ on $S$ vs. $S$ on $R$ [Step (3)], and supramolecular chirality of the molecular network structures, i.e., $\mathrm{CW}$ on $\mathrm{CW}$ vs. CCW on CW [Step (4)].

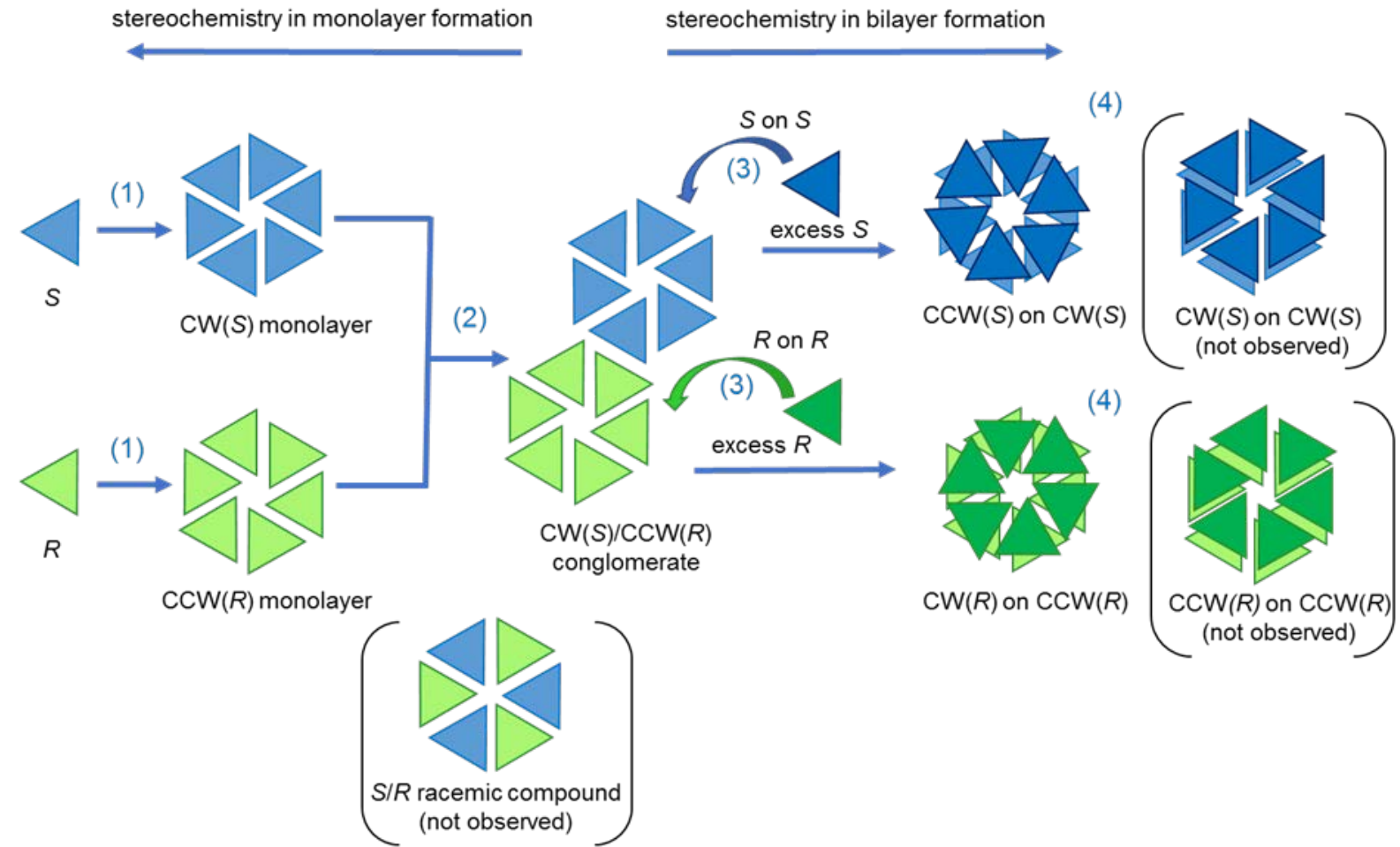

Scheme 2. Schematic of the stereoselectivity in the formation of a self-assembled bilayer of chiral DBA molecules. Blue and green triangles represent cDBA-OC6-OH(S) and cDBAOC6-OH(R), respectively. Chirality arising from the relationship between the orientation of the molecules (or unit cell) with respect to the substrate symmetry axes is not considered. ${ }^{45-47}$ Step (1): Supramolecular enantiospecific self-assembly of cDBA-OC6-OH(S) and cDBAOC6-OH(R) forming clockwise (CW) and counter clockwise (CCW) patterns, respectively (see also Figure 1d). Step (2): Stereoselectivity in a 1:1 mixture of CDBA-OC6-OH(S) and CDBA-OC6-OH(R) forming a domain-separated "conglomerate” consisting of CW and CCW domains; the corresponding "racemic compound" was not observed. Step (3): Diastereospecificity in bilayer formation with respect to the intrinsic chirality of cDBAs in the presence of excess cDBA-OC6-OH(S) or CDBA-OC6-OH(R). The formation of a bilayer with the same handedness, i.e., cDBA-OC6-OH(S) on top of cDBA-OC6-OH(S) or cDBA-OC6$\mathbf{O H}(\boldsymbol{R})$ on top of $\mathbf{c D B A - O C 6 - O H}(\boldsymbol{R})$, is favored. Step (4): Diastereospecificity in bilayer formation with respect to the supramolecular chirality; bilayers consisting of antipodal 
(CW/CCW) patterns of the honeycomb structure are favored over patterns with the same handedness (CW/CW or CCW/CCW).

\section{RESULTS AND DISCUSSSION}

\section{Synthesis of cDBA-OC6-OH(S) and CDBA-OC6-OH(R)}

The synthesis of the homochiral DBA derivatives cDBA-OC6-OH(S) and cDBA-OC6$\mathbf{O H}(\boldsymbol{R})$ is outlined in Scheme 3 for the $S$ enantiomer. We prepared both enantiomers of methoxymethyl (MOM) ethers of 1-bromo-2-hexanol through the selective hydrolytic kinetic resolution of chiral epoxides using a chiral (salen)cobalt(III) catalyst, $^{48}$ followed by regioselective ring opening of the chiral epoxide and MOM protection to insert the hydroxycontaining chiral alkoxy groups into the DBA core. The chiral alkoxy group was introduced to the iodoalkyne building block by coupling the bromide with 3,4-diiodocatechole, followed by monoalkynylation. Copper-catalyzed cyclotrimerization of the iodoalkyne and subsequent deprotection afforded CDBA-OC6-OH(S). Using the same procedure, CDBA-OC6-OH(R) was prepared from the enantiomeric epoxide. The experimental details are described in section 3 of the Supporting Information. The self-assembly of the analytically pure chiral cDBA-OC6OH(S) and cDBA-OC6-OH(R) was analyzed using STM at the interface between highly oriented pyrolytic graphite (HOPG) and one main solvent at various solute concentrations.

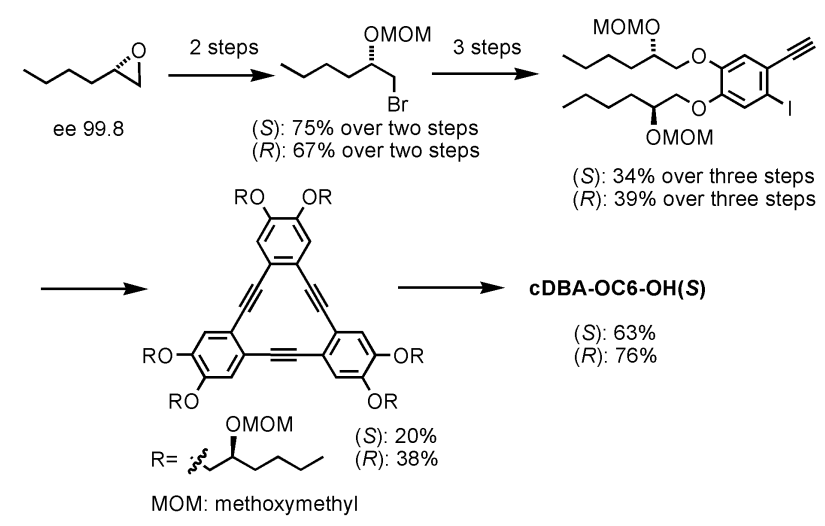

Scheme 3. Outline of the synthesis of CDBA-OC6-OH(S). CDBA-OC6-OH(R) was prepared using the same procedure starting from the enantiomeric epoxide. 
Effects of the Solvent and Concentration on the Formation of a Monolayer and Multilayers

In the first step, we investigated the self-assembly of relatively low concentrations of homochiral enantiomers and racemates of CDBA-OC6-OH(S) and CDBA-OC6-OH(R) at liquid/solid interfaces. Regardless of the solvent [1,2,4-trichlorobenzene (TCB, Figure 1), 1octanol (Figure S1a) or 1-phenyloctane (Figure S1b)], ${ }^{49}$ each enantiomer self-assembles into the typical nanoporous honeycomb networks with opposite 2D chirality, similar to chiral DBAs with stereogenic methyl groups, such as CDBA-OC12(S) and CDBA-OC12(R) (Chart 1). ${ }^{41}$ The DBA cores are lying flat on the substrate and the molecules are linked by van der Waals interactions between the interdigitated alkoxy chains. Figure 1 displays STM images of cDBAOC6-OH(S) and cDBA-OC6-OH(R) at the TCB/HOPG interface, together with a simplified molecular model. The unit cell parameters are $\mathrm{a}=\mathrm{b}=3.2 \mathrm{~nm}$ and the angle $\gamma=60^{\circ}$. These unit cell parameters are identical to the achiral analogue DBA-OC6 without hydroxy groups. ${ }^{44}$ In most STM images, although the alkoxy chains are not clearly visible, the type of interdigitation (+ or -$)$ and the supramolecular chirality [clockwise (CW) or counter clockwise (CCW)] can be determined from the lateral shift of adjacent DBA cores (Figure 1a-d). The overall handedness of the network can also be determined by the signs of the rotation angles of a unit cell vector with respect to one of the symmetry axes of graphite (Figures 1a-b and S2). 


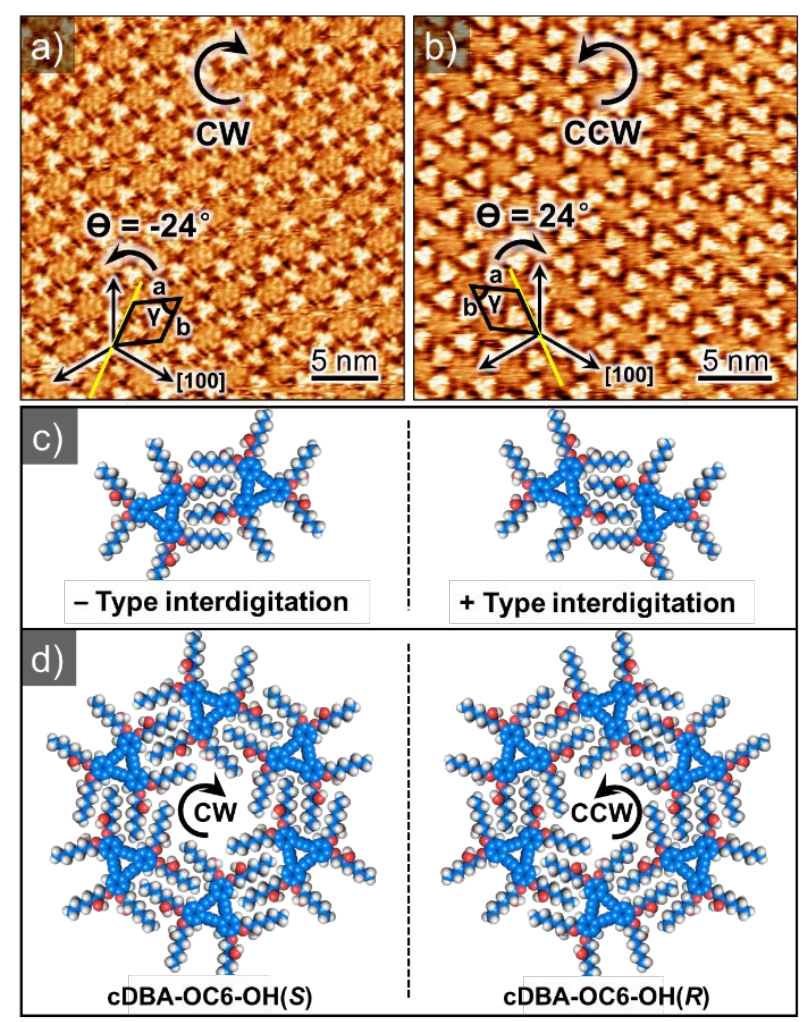

Figure 1. STM images of $\mathbf{C D B A - O C 6 - O H}(S)$ a) and $\mathbf{c D B A - O C 6 - O H}(\boldsymbol{R})$ b) monolayers formed at the TCB/HOPG interface $\left(2.3 \times 10^{-6} \mathrm{M}\right)$. Black arrows indicate the graphite reference axes. $\theta$ is the angle between a reference axis and one of the unit cell vectors. $c$ and d) Molecular models of (-) and (+) interdigitation patterns (c) and corresponding CW and CCW nanowells (d) for CDBA-OC6-OH(S) (a) and CDBA-OC6-OH(R) (b), respectively. Imaging conditions: (a) $I_{\text {set }}=0.2 \mathrm{nA}$ and $V_{\text {bias }}=-0.2 \mathrm{~V}$ and (b) $I_{\text {set }}=0.2 \mathrm{nA}$ and $V_{\text {bias }}=-0.5 \mathrm{~V}$.

Consistent with the stereogenic methyl substitution on the alkyl chains of CDBA-OC12(S) and CDBA-OC12(R), ${ }^{41-42}$ these compounds show enantiomorphous pattern formation. The $S$ enantiomer exclusively produces $\mathrm{CW}$ nanowells via (-) type interdigitation, while the $R$ enantiomer produces $(+)$ interdigitated pairs, leading to the formation of CCW nanowells (Figure 1). Because the supramolecular chirality of the network is the same as CDBA-OC12(S) and $\mathbf{C D B A - O C 1 2}(\boldsymbol{R}),{ }^{41-42}$ the roles of the hydroxy and methyl groups are likely similar in biasing the alkoxy chain interdigitation type and establishing the chirality of the nanowell domains [Step (1) of Scheme 2]. The observed selectivity is consistent with molecular mechanics simulations under periodic boundary conditions (PBC) on a bilayered graphene 
whose geometry was fixed using COMPASS force field. For the $R$ enantiomer, the CCW nanowell formed by the $(+)$ interdigitation type is $1.0 \mathrm{kcal} \mathrm{mol}^{-1}$ more stable than the CW nanowell with the $(-)$ interdigitation type (Figure S3).

Once again, similar to their chiral analogues containing stereogenic methyl groups, ${ }^{41-42}$ when a racemic mixture of CDBA-OC6-OH(S) and cDBA-OC6-OH(R) was deposited at the liquid/solid interface, enantiomorphous domains formed with equal surface coverage. As an example, Figure 2 displays an STM image of racemic cDBA-OC6-OH at the TCB/HOPG interface. Therefore, we conclude that a racemic conglomerate is formed at the liquid/solid interface; both enantiomers assemble in different domains and are phase-separated [Step (2) of Scheme 2]. 


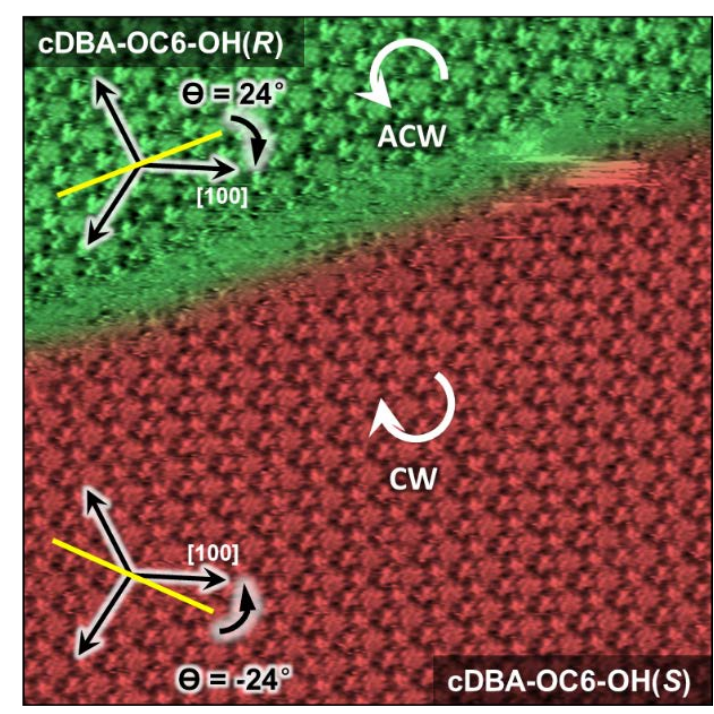

Figure 2. STM image showing the enantiomorphous domains of racemic chiral cDBA-OC6$\mathbf{O H}$ at the TCB/HOPG interface $\left(\mathrm{C}=5.0 \times 10^{-6} \mathrm{M}\right.$, image size $\left.50 \times 50 \mathrm{~nm}^{2}\right)$. In the top left and bottom right panels, cDBA-OC6-OH(R) and cDBA-OC6-OH(S) domains are colored green and red, respectively. The HOPG main symmetry axes are indicated by black arrows. cDBAOC6-OH(S) and CDBA-OC6-OH(R) domains are rotated by $24^{\circ}$ and $-24^{\circ}$ with respect to the surface reference directions (Figure S2). Imaging conditions: $I_{\text {set }}=0.2 \mathrm{nA}$ and $V_{\text {bias }}=-0.2 \mathrm{~V}$.

In contrast to any known DBAs, increasing the concentration of cDBA-OC6-OH leads to noticeable changes in the structure and contrast of the STM images, except when 1-octanol is used as the solvent (Figure S4). Carefully increasing the concentration from $2.3 \times 10^{-6} \mathrm{M}$ to 2.5 $\times 10^{-6} \mathrm{M}$ in $\mathrm{TCB}^{49}$ produces two different types of contrast for the triangular DBA core (Figure 3a and S5). In the higher magnification images (Figure 3b-d), the brighter cDBA molecules are indicated by green dotted triangles. The cDBAs with the same relative position as the brighter cDBA molecules are indicated by solid green triangles, while others are indicated by solid black triangles. From these high-resolution data, the brighter triangle cores of molecules numbered (1), (2) and (3) appear to be rotated by $0^{\circ}, 51^{\circ}$ and $35^{\circ}$ with respect to other reference solid green triangles (Figure 3b-d and S5e-g). A line profile (Figure 3e and S5a, c, and d) shows that the "high" DBA molecules are almost twice as high as the "low” DBA molecules. Based on the height of these nonperiodic protrusions and their varying orientations, we conclude that increasing the concentration led to the adsorption of single molecules in the second layer. 



Figure 3. A high-resolution STM image of cDBA-OC6-OH(S) in TCB (C $\left.=2.5 \times 10^{-6} \mathrm{M}\right)$ reveals isolated molecules on top of the bottom layer. a) Large scale image (53 $\left.\times 53 \mathrm{~nm}^{2}\right)$. b-c) Digital magnification of the image shown in (a). A full scale image of (d) is presented in Figure S5b. b-d) Green dotted triangles indicate the orientation of the bright DBAs. The cDBAs with the same relative position as the brighter cDBAs are indicated by solid green triangles, while others are indicated by solid black triangles. e) Line profile across a "high” DBA molecule, as indicated by the green line in panel (a). a-d) Imaging conditions: $I_{\text {set }}=0.2 \mathrm{nA}$ and $V_{\text {bias }}=-1.4$ V.

A further increase in the concentration to $2.6 \times 10^{-6} \mathrm{M}$ increases the density of molecules in the second layer (Figure 4), with the formation of on-top dimers. Interestingly, the relative orientation of the triangles in this on-top dimer (Figure $4 \mathrm{~b}$ and c) differs from the relative orientation of adjacent molecules one layer below ((-) type interdigitation, Figure $4 \mathrm{~d})$. In the former case, maximum overlap is observed between the two adjacent sides of neighboring triangles, as opposed to the apparent shift of the molecules in the layer underneath. 


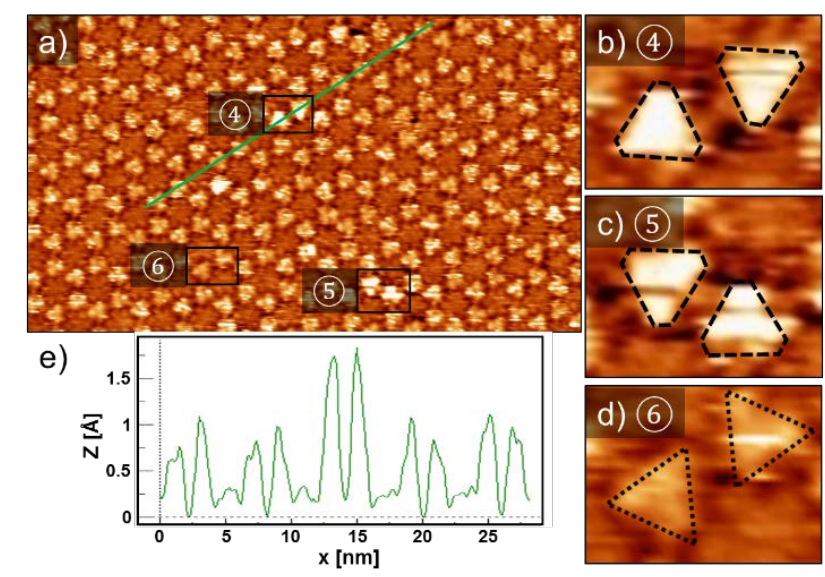

Figure 4. High-resolution STM image of cDBA-OC6-OH(S) in TCB revealing the initial formation of second layer dimers. a) Large scale image (42 $\times 24 \mathrm{~nm}^{2}$; C $\left.=2.6 \times 10^{-6} \mathrm{M}\right)$. b-d) Digital magnification of the image shown in (a). The triangle cores of the second layer dimer (b and c) appear to face each other as opposed to being shifted for monolayer dimers (d). Imaging condition: $I_{\text {set }}=0.2 \mathrm{nA}$ and $V_{\text {bias }}=-1.4 \mathrm{~V}$. e) Line profile across a second layer dimer, as indicated by the green line in panel (a).

A further increase in the concentration $\left(2.7 \times 10^{-6} \mathrm{M}\right)$ also leads to the formation of a more extended second layer (Figure S6a). Actually, the second layer appears to be adsorbed so strongly to the first layer that it is unable to easily be removed by rinses with the solvent (Figure S6b). This finding differs from the SAMN formed from DBA-OC6, which does not show any multilayer growth, even at a very high solution concentration of $1.2 \times 10^{-2} \mathrm{M}$ (Figure S7). For related compounds carrying a methyl group on the chiral center, CDBA-OC12(S) and cDBA$\operatorname{OC12}(\boldsymbol{R})$, multilayer formation was never observed in the same solvents. ${ }^{41}$

A partial double layer was also observed in a 1-phenyloctane solution for CDBA-OC6-OH (Figure S8), while regardless of the concentration, only monolayer formation was observed in 1-octanol (Figure S4). These results support but do not validate our hypothesis that longitudinal hydrogen bonding promotes the growth of a second layer.

\section{Bias Voltage Dependence of Monolayer versus Multilayers Imaging}


At a high concentration of the chiral DBA $\left(>2.9 \times 10^{-6} \mathrm{M}\right)$ in TCB, the image contrast depends on the imaging conditions. For example, the two sequential images (taken 2 min apart) in Figure 5a-b for cDBA-OC6-OH(R) $\left(1 \times 10^{-3} \mathrm{M}\right)$ at the TCB/HOPG interface clearly display the effect of the bias voltage. Figure $5 \mathrm{a}$ was recorded at the bias voltage ( $\left.V_{\text {bias }}\right)$ of $-0.5 \mathrm{~V}$, whereas Figure $5 \mathrm{~b}$ was recorded at $V_{\text {bias }}$ of $-1.2 \mathrm{~V}$. The SAMN in Figure $5 \mathrm{a}$ contains narrow domain boundaries, as indicated by white dashed lines, which are typically less than one molecule wide. The STM image recorded at $-1.2 \mathrm{~V}$ (Figure $5 \mathrm{~b}$ ) displays a remarkable difference in contrast, showing some dark trenches that appear to coincide with the white lines in Figure 5a to some extent. We tentatively interpret the difference in contrast at the more negative sample bias to the visualization of the top layer of a multilayer structure. Additional higher magnification images in Figure 5c with the respective height profiles (Figure 5d) at large negative sample bias ( $V_{\text {bias }}=-1.1 \mathrm{~V}$ ) further support the formation of double layers. The line profile (Figure 5d) shows a significant difference in height between the "low" and "high" DBA molecules, which supports the interpretation that the "high" molecules are part of the top of a double layer or multilayer. Figure 5 c reveals details of the “dark trenches”: the multilayers do not readily grow at the domain boundaries. 

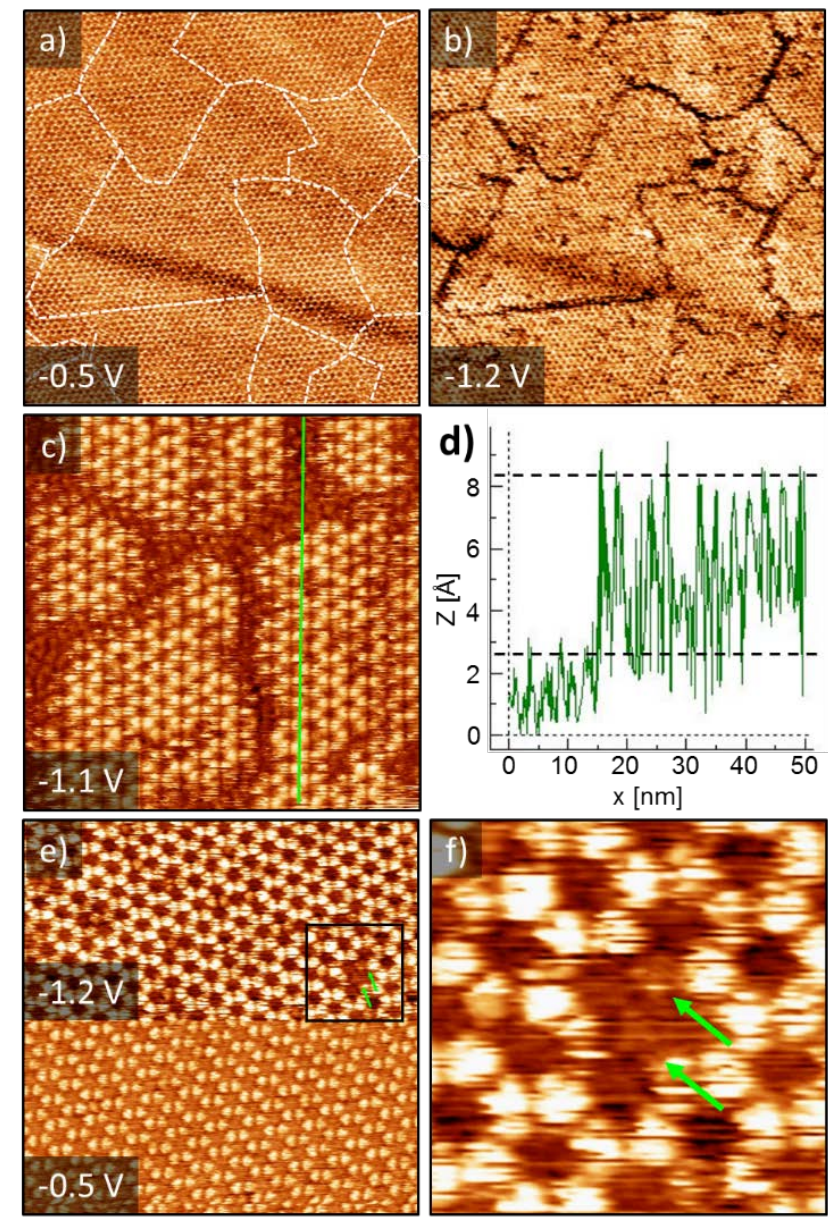

Figure 5. STM image of cDBA-OC6-OH(S) in TCB $\left(1 \times 10^{-3} \mathrm{M}\right)$ showing the effect of the bias voltage on the contrast of the multilayer structure. a and b) Sequential STM images (170 $\times 170 \mathrm{~nm}^{2}$ ) captured at a bias voltage of $-0.5 \mathrm{~V}$ (panel (a) showing monolayer) and $-1.2 \mathrm{~V}$ (panel (b) showing double layer with dark trenches), respectively. White dashed lines in (a) indicate domain borders. c) A higher magnification image $\left(55 \times 55 \mathrm{~nm}^{2}\right)$ showing more details of the dark trenches captured at sample bias voltage $-1.1 \mathrm{~V}$. d) Line profiles along the green lines indicated in (c). e) In the middle of the image, the voltage increased from $-1.2 \mathrm{~V}$ (showing double layer) to $-0.5 \mathrm{~V}$ (showing monolayer) $\left(50 \times 50 \mathrm{~nm}^{2}\right)$ from top to bottom. In the top half of the image, defects in the top layer (marked by green arrows in panel (e) and its higher magnification image in panel (f)) allow us to visualize molecules in the lower layer, which appear darker than the surrounding molecules. For all the images: $I_{\text {set }}=0.2 \mathrm{nA}$. 
By switching the bias voltage during scanning, the imaging mode is able to be switched reversibly between multiple and single layers (Figure 5e). During scanning, the voltage was increased from $-1.2 \mathrm{~V}$ to $-0.5 \mathrm{~V}$. As a result, the top half of the image appears brighter than the bottom half, consistent with the imaging of a multilayer at larger negative sample bias and of a monolayer at smaller negative bias. Similar bias-voltage dependences of monolayer and multilayers have been reported for some other systems. ${ }^{16,20,37,50}$ Upon decreasing the absolute value of the voltage, the tip-substrate distance decreases, and the STM tip likely penetrates through the double layer to image the bottom layer (see the SI, including Figure S12, for more information).

\section{Stereospecificity in Bilayer Formation}

At a low concentration, a racemic solution of $\mathbf{~ C D B A - O C 6 - O H ~ ( t o t a l ~ c o n c e n t r a t i o n s ~ o f ~} 5 \times$ $10^{-6} \mathrm{M}$ for TCB (Figure 2) and $2 \times 10^{-5} \mathrm{M}$ for 1-phenyloctane (Figure S9)) self-assembles into a domain-separated conglomerate consisting of domains of each enantiomer. Similarly, a racemate at high concentration $\left(>1 \times 10^{-4} \mathrm{M}\right.$ ) also tends to form a domain-separated bilayer (Figure S10). We investigated if bilayer formation is diastereospecific in terms of (i) the intrinsic chirality of cDBAs in the first and second layers [Step (3) in Scheme 2] and (ii) supramolecular chirality, i.e., CW/CCW patterns of the honeycomb structure, of the first and second layers [Step (4) in Scheme 2].

First, we prepared a low concentration, equimolar solution of both enantiomers in TCB and placed $20 \mu \mathrm{L}$ of this solution in a liquid cell with the HOPG substrate. After network formation was verified using STM, an excess amount of one of the enantiomers, CDBA-OC6-OH(R) in the case of Figure 6, was added to the liquid cell. This sequential addition protocol ensures that the domains of both enantiomers initially formed. STM imaging at a small negative bias of 0.6 V clearly revealed the formation of separate CW and CCW domains in the first layer (Figure 6a), similar to STM images of monolayers formed at a low concentration (Figure 2). The domains in the upper and lower half of Figure 6a are attributed to $\mathbf{C D B A - O C 6 - O H}(\boldsymbol{R})$ and cDBA-OC6-OH(S) domains, respectively, based on the signs of the domain rotation angles with respect to one of the symmetry axes of graphite (Figures 2 and S11). By decreasing the 
bias voltage from $-0.6 \mathrm{~V}$ to $-1.2 \mathrm{~V}$, a second layer is observed exclusively on top of the CCW domain of cDBA-OC6-OH(R) (Figure 6b). Given the excess amount of cDBA-OC6-OH(R) in solution, the exclusive observation of the double layer on a CCW domain indicates that the top layer also consists of cDBA-OC6-OH(R). Experiments in which an excess of cDBA-OC6$\mathbf{O H}(\boldsymbol{S})$ was added produced similar results, as shown in Figure S13. In the latter case, bilayer formation is only observed on CW domains. When using a premixed solution of both enantiomers with an excess of one of the enantiomers, a second layer is only observed on top of the domains formed by the major enantiomer, consistent with the results obtained upon sequential addition (Figure S14).
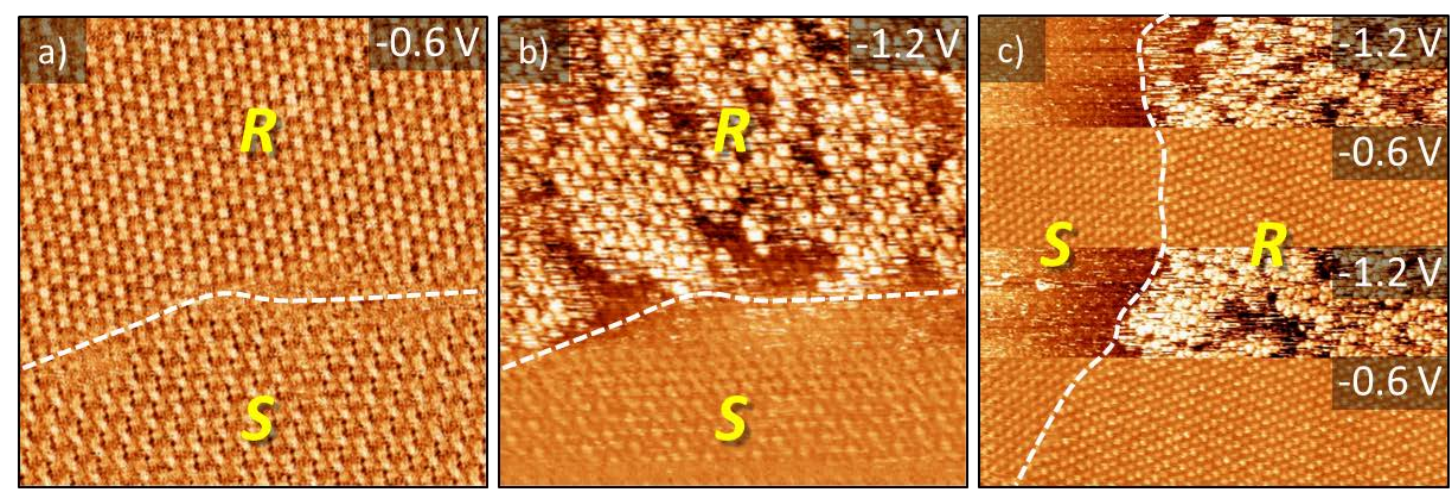

Figure 6. Enantioselective formation of a multilayer structure. The sequential addition of a 1:1 mixture of CDBA-OC6-OH(S) and CDBA-OC6-OH(R) (concentration of each enantiomer = 3 $\left.\times 10^{-4} \mathrm{M}, 20 \mu \mathrm{L}\right)$, followed by the addition of $\mathbf{C D B A - O C 6 - O H}(\boldsymbol{R})\left(1.2 \times 10^{-3} \mathrm{M}, 20 \mu \mathrm{L}\right)$ in a liquid cell at the TCB/HOPG interface. (a - c) Images were captured after excess cDBA-OC6$\mathbf{O H}(\boldsymbol{R})$ was added. White dashed lines indicate domain boundaries. a and b) Sequential images captured at the same location. a) Image of the bottom layer at $-0.6 \mathrm{~V}$. The top domain is determined to be cDBA-OC6-OH(R) and the bottom domain is determined to be CDBA-OC6$\mathbf{O H}(\mathbf{S})$. For more details, see Figure S11. b) At $-1.2 \mathrm{~V}$, double layer formation was observed only on top of the CDBA-OC6-OH(R) domain. c) The STM image obtained by modulating the bias voltage during the scanning process. a-c) Image size $100 \times 100 \mathrm{~nm}^{2} . I_{\text {set }}=0.2 \mathrm{nA}$ and $V_{\text {bias }}$ are indicated in the image.

In addition to the domain-dependent layer growth, we are also able to monitor each layer separately, depending on the chosen STM bias voltage. The contrast switch in the image occurs instantaneously as the bias voltage is modulated. In Figure 6c, double layer formation is 
observed at $-1.2 \mathrm{~V}$, while the underlying bottom layer is revealed at $-0.6 \mathrm{~V}$. This approach allows us to determine the handedness of the bottom layer and confirms that in the presence of excess CDBA-OC6-OH(R), bilayer formation is only observed on top of the CCW bottom layer domains. Figure S13 illustrates this principle for excess cDBA-OC6-OH(S). When a racemic solution of cDBAs at relatively high concentration was used, domain-separated bilayers consisting of homochiral domains of cDBA were observed, although we did not determine their stereochemistry (Figure S10).

No evidence is found that concentration-dependent aggregation in solution is involved in the formation of these structures. In a $\mathrm{CDCl}_{3}$ solution, $\mathrm{cDBAs}$ exhibit a small concentrationdependent ${ }^{1} \mathrm{H}$ NMR chemical shift, suggesting weak aggregation (see Supporting Information section 5). However, this shift is nearly identical in homochiral and racemate solutions, refuting the idea of stereospecific association in solution. The enantioselective formation of the double layer structure with respect to the intrinsic chirality of cDBA is therefore concluded to be a specific phenomenon occurring at the interface between the solid HOPG support and the solution on top.

Next, we investigated if bilayer formation is diastereospecific with respect to supramolecular chirality of the first and second layers [Step (4), Scheme 2]. For example, for the $S$ enantiomer, the first layer adopts the CW honeycomb pattern through the (-) type interdigitation of the alkoxy chains (Figure 1a). Depending on whether the second layer adopts the antipodal CCW pattern or the same-handed CW pattern, the double layer will be diastereomeric with respect to supramolecular chirality [Step (4) in Scheme 2]. Homochiral solutions were used to probe the supramolecular chirality of the second layer while excluding the possibility of interference from the DBA molecules with the opposite chirality. Figure 7a shows an STM image of cDBA-OC6-OH(S) $\left(1 \times 10^{-3} \mathrm{M}\right)$ at the TCB/HOPG interface. The triangular features, which are highlighted by the black dotted lines, form a pattern with $p 6 m$ plane group symmetry, as opposed to the chiral $p 6$ symmetry of the monolayer (Figure 7a, inset). ${ }^{51}$ The appearance of this apparent achiral feature is potentially explained in terms of offset of shifts between overlapping antipodal triangles, as shown in Figure 7b. This type of staggered packing was observed for the crystal packing of other DBA derivatives (achiral), as evidenced by single crystal structures. ${ }^{52-53}$ Accordingly, we deduce that the second layer adopts an antipodal supramolecular chirality, i.e., CCW on CW and CW on CCW, for cDBA-OC6$\mathbf{O H}(S)$ and cDBA-OC6-OH(R), respectively. 


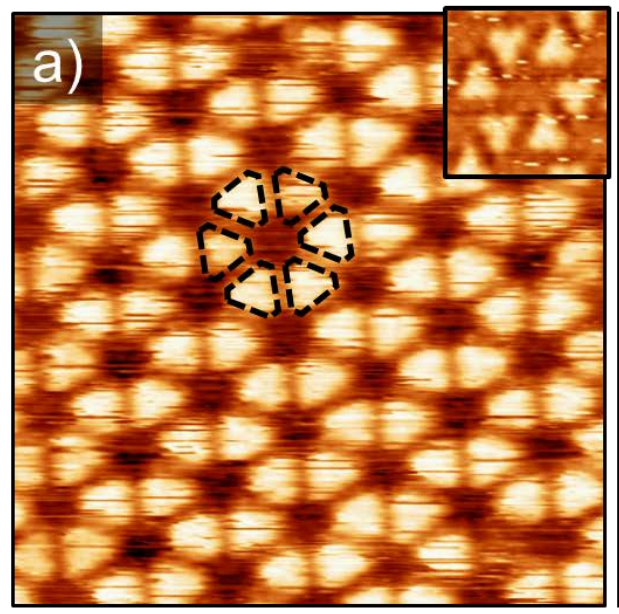

b) Double layer formation

Dimers

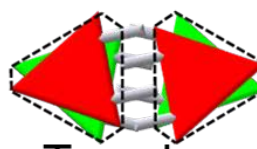

Top view

III

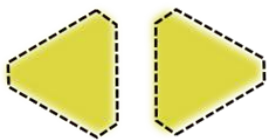

STM view

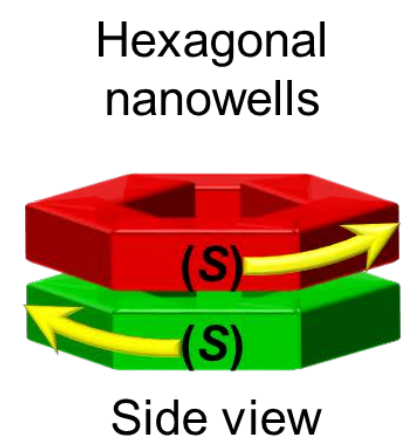

Figure 7. a) STM image of the self-assembly of a multilayered cDBA-OC6-OH(S) structure $\left(1 \times 10^{-3} \mathrm{M}\right)$ at the TCB/HOPG interface $\left(20 \times 20 \mathrm{~nm}^{2}\right)$. The black dashed triangles indicate the apparent orientations of the triangle DBA cores of the multilayer. Inset: Monolayer selfassembly of cDBA-OC6-OH(S). Note the change in the plane group from chiral $p 6$ to achiral p6m for the monolayer (inset) and double layer (panel (a)), respectively. b) Proposed multilayer growth model where the bottom layer and the second layer have opposite interdigitation patterns. At the dimer level for $\mathbf{c D B A - O C 6 - O H}(\boldsymbol{S})$, the bottom layer (green) adopts the (-) interdigitation pattern (see Figure 1), while the second layer (red) adopts the $(+)$ interdigitation pattern. At the supramolecular hexagonal nanowell level, the bottom layer (green) exhibits the CW pattern (see Figure 1), while the second layer (red) displays the CCW pattern. The red and green triangle cores are right on top of each other but rotated. The STM contrast shows a superimposed image of two rotated DBA triangle cores in the first and second layer, as outlined by the dotted triangles. Imaging conditions: $I_{\mathrm{set}}=0.2 \mathrm{nA}$ and $V_{\text {bias }}=-1.4 \mathrm{~V}$.

A series of molecular mechanics (MM) optimizations using COMPASS force field were performed to obtain insights into the molecular structure of and intermolecular interactions in different bilayers. Table S1 shows the lowest energy local minima we observed for bilayers composed of cDBA-OC6-OH(R) and cDBA-OC6-OH(R)/CDBA-OC6-OH(S) (Figure S15). When both layers have the same supramolecular chirality (e.g., CCW/CCW or Figure S15a), their DBA cores are laterally shifted with respect to each other, and when the layers differ in supramolecular chirality (e.g., CCW/CW or Figure S15c), their DBA cores are rotated with respect to each other. This finding forms the basis for the assignment of chirality of the layers in STM images of different bilayers (Figure S15). The MM optimizations also reveal that certain $\mathrm{OH}$ groups are capable of forming hydrogen bonds $(\mathrm{OH} \cdots \mathrm{O}$ distances ranging between 
ca. 1.7 and $2.1 \AA$, Table S2), providing additional stabilization to bilayer structures. The estimated energy values (Table S1) are not consistent with the experimental results. This latter point is not surprising considering the conformational flexibility in these systems and our neglect of the role of the solvent. In addition, kinetic factors may overrule thermodynamic equilibrium. Indeed, the annealing of a solution of CDBA-OC6-OH(R) in TCB on HOPG at $80{ }^{\circ} \mathrm{C}$ for $10 \mathrm{~min}$ resulted in the formation of a new phase, the trimer phase (Figure S16). ${ }^{38}$ This result indicates the complexity of the system, where the multilayer is a metastable or a kinetically trapped phase. A further detailed analysis of the stability of the multilayer vs other phases is required to understand the thermodynamics of the present complicated system.

\section{CONCLUSIONS}

For the first time, we were able to achieve the stereo-controlled growth of a multilayered supramolecular porous network at a liquid/solid interface. DBA derivatives bearing alkoxy chains substituted at the 2-position with a hydroxy group were designed to extend the growth towards the third dimension. Upon forming a self-assembled monolayer, three hydroxy groups are rotated out-of-plane away from the surface to potentially facilitate hydrogen bonding interactions with the overlaying layer. Under appropriate concentration and solvent conditions, double layer formation was confirmed by bias voltage-dependent STM observations. Both lateral van der Waals interactions and possibly also longitudinal hydrogen bonds contribute to the formation of multilayers. Based on the experimental evidence, the formation of homochiral double layers, i.e., bilayer formation, is a stereospecific process, and the top and bottom layer have the same intrinsic chirality. Surprisingly, the overlaying layer adopts opposite supramolecular handedness with respect to that of the first layer, highlighting the complexity of self-assembly pathways and the mechanisms involved in the amplification of chirality.

\section{ASSOCIATED CONTENT}

\section{Supporting Information}

The Supporting Information is available free of charge at hppts://pubs.acs.org/doi/......

Experimental details, MM Simulations, additional STM images, and synthesis of chiral DBA derivatives (PDF) 


\section{AUTHOR INFORMATION}

\section{Corresponding Author}

* steven.defeyter@kuleuven.be

* tobe@chem.es.osaka-u.ac.jp

\section{ORCID IDs}

Yoshito Tobe: 0000-0002-1795-5829

Steven De Feyter: 0000-0002-0909-9292

Kazukuni Tahara: 0000-0002-3634-541X

Dmitrii Perepichka: 0000-0003-2233-416X

Rustam Z. Khaliullin: 0000-0002-9073-6753

Zhenzhe Zhang: 0000-0002-9983-7729

Yuan Fang: 0000-0002-4846-1725

\section{Notes}

The authors declare no competing financial interests.

\section{ACKNOWLEDGMENTS}

This study was supported by JSPS KAKENHI (JP15H02164 and JP18H05353 for Y.T. and JP17K19130 and JP19H04597 for K.T.) and by Center for Emergent Functional Matter Science, National Chiao Tung University, which are gratefully acknowledged. The authors also acknowledge financial support from the Fund of Scientific Research Flanders (FWO) under grant EOS 30489208 and KU Leuven - Internal Funds. The research leading to these results has also received funding from the European Research Council under the European Union's Seventh Frame-work Programme (FP7/2007-2013)/ERC Grant Agreement No. 340324 to S.D.F. The work in Canada was supported by NSERC Discovery Grants (to R.K. and to D.F.P.).

\section{REFERENCES}

1. Goronzy, D. P.; Ebrahimi, M.; Rosei, F.; Arramel; Fang, Y.; De Feyter, S.; Tait, S. L.; Wang, C.; Beton, P. H.; Wee, A. T. S.; Weiss, P. S.; Perepichka, D. F., Supramolecular 
Assemblies on Surfaces: Nanopatterning, Functionality, and Reactivity. ACS Nano 2018, 12, 7445-7481.

2. Sosa-Vargas, L.; Kim, E.; Attias, A.-J., Beyond “decorative” 2D supramolecular selfassembly: strategies towards functional surfaces for nanotechnology. Mater Horiz. 2017, 4, 570-583.

3. Mali, K. S.; Pearce, N.; De Feyter, S.; Champness, N. R., Frontiers of supramolecular chemistry at solid surfaces. Chem. Soc. Rev. 2017, 46, 2520-2542.

4. Bonifazi, D.; Mohnani, S.; Llanes-Pallas, A., Supramolecular Chemistry at Interfaces: Molecular Recognition on Nanopatterned Porous Surfaces. Chem. Eur. J. 2009, 15, 7004-7025. 5. Ma, X.; Yang, Y.; Deng, K.; Zeng, Q.; Zhao, K.; Wang, C.; Bai, C., Molecular miscibility characteristics of self-assembled 2D molecular architectures. J. Mater. Chem. 2008, 18, 2074-2081.

6. Iritani, K.; Tahara, K.; De Feyter, S.; Tobe, Y., Host-Guest Chemistry in Integrated Porous Space Formed by Molecular Self-Assembly at Liquid-Solid Interfaces. Langmuir 2017, 33, 4601-4618.

7. Stepanow, S.; Lingenfelder, M.; Dmitriev, A.; Spillmann, H.; Delvigne, E.; Lin, N.; Deng, X.; Cai, C.; Barth, J. V.; Kern, K., Steering molecular organization and host-guest interactions using two-dimensional nanoporous coordination systems. Nat. Mater. 2004, 3, 229233.

8. Heinke, L.; Gu, Z.; Woell, C., The surface barrier phenomenon at the loading of metalorganic frameworks. Nat. Commun. 2014, 5, 4562.

9. Liu, J.; Zhou, W.; Liu, J.; Howard, I.; Kilibarda, G.; Schlabach, S.; Coupry, D.; Addicoat, M.; Yoneda, S.; Tsutsui, Y.; Sakurai, T.; Seki, S.; Wang, Z.; Lindemann, P.; Redel, E.; Heine, T.; Wöll, C., Photoinduced Charge-Carrier Generation in Epitaxial MOF Thin Films: High Efficiency as a Result of an Indirect Electronic Band Gap? Angew. Chem. Int. Ed. 2015, 54, 7441-7445.

10. Ciesielski, A.; Samori, P., Supramolecular Approaches to Graphene: From SelfAssembly to Molecule-Assisted Liquid-Phase Exfoliation. Adv. Mater. 2016, 28, 6030-6051.

11. Zheng, Q. N.; Liu, X. H.; Chen, T.; Yan, H. J.; Cook, T.; Wang, D.; Stang, P. J.; Wan, L. J., Formation of Halogen Bond-Based 2D Supramolecular Assemblies by Electric Manipulation. J. Am. Chem. Soc. 2015, 137, 6128-6131.

12. Dutta, S.; Gellman, A. J., Enantiomer surface chemistry: conglomerate versus racemate formation on surfaces. Chem. Soc. Rev. 2017, 46, 7787-7839.

13. Samori, P.; Severin, N.; Simpson, C. D.; Mullen, K.; Rabe, J. P., Epitaxial composite layers of electron donors and acceptors from very large polycyclic aromatic hydrocarbons. $J$. Am. Chem. Soc. 2002, 124, 9454-9457.

14. Samorí, P.; Fechtenkötter, A.; Jäckel, F.; Böhme, T.; Müllen, K.; Rabe, J. P., Supramolecular Staircase via Self-Assembly of Disklike Molecules at the Solid-Liquid Interface. J. Am. Chem. Soc. 2001, 123, 11462-11467. 
15. Dossel, L. F.; Kamm, V.; Howard, I. A.; Laquai, F.; Pisula, W.; Feng, X.; Li, C.; Takase, M.; Kudernac, T.; De Feyter, S.; Mullen, K., Synthesis and controlled self-assembly of covalently linked hexa-peri-hexabenzocoronene/perylene diimide dyads as models to study fundamental energy and electron transfer processes. J. Am. Chem. Soc. 2012, 134, 5876-5886. 16. Piot, L.; Marie, C.; Feng, X.; Muellen, K.; Fichou, D., Hierarchical Self-Assembly of Edge-On Nanocolumnar Superstructures of Large Disc-Like Molecules. Adv. Mater. 2008, 20, 3854-3858.

17. Huang, Y. L.; Chen, W.; Wee, A. T. S., Molecular Trapping on Two-Dimensional Binary Supramolecular Networks. J. Am. Chem. Soc. 2011, 133, 820-825.

18. Yoshimoto, S.; Tsutsumi, E.; Suto, K.; Honda, Y.; Itaya, K., Molecular assemblies and redox reactions of zinc(II) tetraphenylporphyrin and zinc(II) phthalocyanine on Au(111) single crystal surface at electrochemical interface. Chem. Phys. 2005, 319, 147-158.

19. Uemura, S.; Sakata, M.; Taniguchi, I.; Hirayama, C.; Kunitake, M., In situ observation of coronene epitaxial adlayers on $\mathrm{Au}(111)$ surfaces prepared by the transfer of Langmuir films. Thin Solid Films 2002, 409, 206-210.

20. Ivasenko, O.; MacLeod, J. M.; Chernichenko, K. Y.; Balenkova, E. S.; Shpanchenko, R. V.; Nenajdenko, V. G.; Rosei, F.; Perepichka, D. F., Supramolecular assembly of heterocirculenes in 2D and 3D. Chem. Commun. 2009, 1192-1194.

21. Ikeda, T.; Asakawa, M.; Goto, M.; Miyake, K.; Ishida, T.; Shimizu, T., STM Observation of Alkyl-Chain-Assisted Self-Assembled Monolayers of Pyridine-Coordinated Porphyrin Rhodium Chlorides. Langmuir 2004, 20, 5454-5459.

22. Skomski, D.; Jo, J.; Tempas, C. D.; Kim, S.; Lee, D.; Tait, S. L., High-Fidelity SelfAssembly of Crystalline and Parallel-Oriented Organic Thin Films by $\pi-\pi$ Stacking from a Metal Surface. Langmuir 2014, 30, 10050-10056.

23. Niederhausen, J.; Kersell, H. R.; Christodoulou, C.; Heimel, G.; Wonneberger, H.; Mullen, K.; Rabe, J. P.; Hla, S. W.; Koch, N., Monolayer Phases of a Dipolar Perylene Derivative on $\mathrm{Au}(111)$ and Surface Potential Build-Up in Multilayers. Langmuir 2016, 32, 3587-600.

24. Pivetta, M.; Blüm, M.-C.; Patthey, F.; Schneider, W.-D., Three-Dimensional Chirality Transfer in Rubrene Multilayer Islands on Au(111). J. Phys. Chem. B 2009, 113, 4578-4581. 25. Mairena, A.; Zoppi, L.; Seibel, J.; Tröster, A. F.; Grenader, K.; Parschau, M.; Terfort, A.; Ernst, K.-H., Heterochiral to Homochiral Transition in Pentahelicene 2D Crystallization Induced by Second-Layer Nucleation. ACS Nano 2017, 11, 865-871.

26. Parschau, M.; Ernst, K. H., Disappearing Enantiomorphs: Single Handedness in Racemate Crystals. Angew. Chem. Int. Ed. 2015, 54, 14422-14426.

27. Parschau, M.; Ellerbeck, U.; Ernst, K.-H., Chirality transfer by epitaxial mismatch in multi-layered homochiral molecular films. Colloids Surf. A 2010, 354, 240-245.

28. Racemate and conglomerate double layers have opposite or the same chirality between layers, respectively. 
29. Cao, H.; De Feyter, S., Amplification of chirality in surface-confined supramolecular bilayers. Nat. Commun. 2018, 9, 3416-3416.

30. Lee, S.; Hirsch, B. E.; Liu, Y.; Dobscha, J. R.; Burke, D. W.; Tait, S. L.; Flood, A. H., Multifunctional Tricarbazolo Triazolophane Macrocycles: One-Pot Preparation, Anion Binding, and Hierarchical Self-Organization of Multilayers. Chem. Eur. J. 2016, 22, 560-569.

31. Svatek, S. A.; Perdigao, L. M. A.; Stannard, A.; Wieland, M. B.; Kondratuk, D. V.; Anderson, H. L.; O'Shea, J. N.; Beton, P. H., Mechanical Stiffening of Porphyrin Nanorings through Supramolecular Columnar Stacking. Nano Lett. 2013, 13, 3391-3395.

32. Huang, W.; Zhao, T.-Y.; Wen, M.-W.; Yang, Z.-Y.; Xu, W.; Yi, Y.-P.; Xu, L.-P.; Wang, Z.-X.; Gu, Z.-J., Adlayer Structure of Shape-Persistent Macrocycle Molecules: Fabrication and Tuning Investigated with Scanning Tunneling Microscopy. J. Phys. Chem. C 2014, 118, 6767-6772.

33. Iritani, K.; Ikeda, M.; Yang, A.; Tahara, K.; Hirose, K.; Moore, J. S.; Tobe, Y., Hexagonal Molecular Tiling by Hexagonal Macrocycles at the Liquid/Solid Interface: Structural Effects on Packing Geometry. Langmuir 2017, 33, 12453-12462.

34. Hirsch, B. E.; Lee, S.; Qiao, B.; Chen, C. H.; McDonald, K. P.; Tait, S. L.; Flood, A. H., Anion-induced dimerization of 5-fold symmetric cyanostars in 3D crystalline solids and 2D self-assembled crystals. Chem. Commun. 2014, 50, 9827-9830.

35. Wieland, M. B.; Perdigao, L. M. A.; Kondratuk, D. V.; O'Shea, J. N.; Anderson, H. L.; Beton, P. H., Height dependent molecular trapping in stacked cyclic porphyrin nanorings. Chem. Commun. 2014, 50, 7332-7335.

36. Ciesielski, A.; Cadeddu, A.; Palma, C.-A.; Gorczynski, A.; Patroniak, V.; Cecchini, M.; Samori, P., Self-templating 2D supramolecular networks: a new avenue to reach control over a bilayer formation. Nanoscale 2011, 3, 4125-4129.

37. Blunt, M. O.; Russell, J. C.; Gimenez-Lopez, M. d. C.; Taleb, N.; Lin, X.; Schroder, M.; Champness, N. R.; Beton, P. H., Guest-induced growth of a surface-based supramolecular bilayer. Nat. Chem. 2011, 3, 74-78.

38. Tahara, K.; Furukawa, S.; Uji-i, H.; Uchino, T.; Ichikawa, T.; Zhang, J.; Mamdouh, W.; Sonoda, M.; De Schryver, F. C.; De Feyter, S.; Tobe, Y., Two-Dimensional Porous Molecular Networks of Dehydrobenzo[12]annulene Derivatives via Alkyl Chain Interdigitation. J. Am. Chem. Soc. 2006, 128, 16613-16625.

39. Tobe, Y.; Tahara, K.; De Feyter, S., Adaptive Building Blocks Consisting of Rigid Triangular Core and Flexible Alkoxy Chains for Self-Assembly at Liquid/Solid Interfaces. Bull. Chem. Soc. Jpn. 2016, 89, 1277-1306.

40. Lei, S. B.; Tahara, K.; De Schryver, F. C.; Van der Auweraer, M.; Tobe, Y.; De Feyter, S., One building block, two different supramolecular surface-confined patterns: Concentration in control at the solid-liquid interface. Angew. Chem. Int. Ed. 2008, 47, 2964-2968. 
41. Tahara, K.; Yamaga, H.; Ghijsens, E.; Inukai, K.; Adisoejoso, J.; Blunt, M. O.; De Feyter, S.; Tobe, Y., Control and induction of surface-confined homochiral porous molecular networks. Nat. Chem. 2011, 3, 714-719.

42. Fang, Y.; Ghijsens, E.; Ivasenko, O.; Cao, H.; Noguchi, A.; Mali, K. S.; Tahara, K.; Tobe, Y.; De Feyter, S., Dynamic control over supramolecular handedness by selecting chiral induction pathways at the solution-solid interface. Nat. Chem. 2016, 8, 711-717.

43. Tahara, K.; Noguchi, A.; Nakayama, R.; Ghijsens, E.; De Feyter, S.; Tobe, Y., Reversing the Handedness of Self-Assembled Porous Molecular Networks through the Number of Identical Chiral Centres. Angew. Chem. Int. Ed. 2019, 58, 7733-7738.

44. Balandina, T.; Tahara, K.; Sandig, N.; Blunt, M. O.; Adisoejoso, J.; Lei, S. B.; Zerbetto, F.; Tobe, Y.; De Feyter, S., Role of Substrate in Directing the Self-Assembly of Multicomponent Supramolecular Networks at the Liquid-Solid Interface. Acs Nano 2012, 6, 8381-8389.

45. Barlow, S. M.; Raval, R., Complex organic molecules at metal surfaces: bonding, organisation and chirality. Surf. Sci. Rep. 2003, 50, 201-341.

46. Elemans, J.; De Cat, I.; Xu, H.; De Feyter, S., Two-dimensional chirality at liquidsolid interfaces. Chem. Soc. Rev. 2009, 38, 722-736.

47. Raval, R., Chiral expression from molecular assemblies at metal surfaces: insights from surface science techniques. Chem. Soc. Rev. 2009, 38, 707-721.

48. Yoon, T. P.; Jacobsen, E. N., Privileged Chiral Catalysts. Science 2003, 299, 16911693.

49. All the data shown in the main text are done in a liquid cell using $40 \mu \mathrm{L}$ of solution. Similar results can be obtained by simple drop casting method using $4 \mu \mathrm{L}$ of solution (more detail see supporting information). Large quantity of solution are used to minimize the concentration change due to evaporation.

50. Crivillers, N.; Furukawa, S.; Minoia, A.; Heyen, A. V.; Mas-Torrent, M.; Sporer, C.; Linares, M.; Volodin, A.; Van Haesendonck, C.; Van der Auweraer, M.; Lazzaroni, R.; De Feyter, S.; Veciana, J.; Rovira, C., Two-Leg Molecular Ladders Formed by Hierarchical SelfAssembly of an Organic Radical. J. Am. Chem. Soc. 2009, 131, 6246-6252.

51. Plass, K. E.; Grzesiak, A. L.; Matzger, A. J., Molecular packing and symmetry of twodimensional crystals. Acc. Chem. Res. 2007, 40, 287-293.

52. Hisaki, I.; Senga, H.; Sakamoto, Y.; Tsuzuki, S.; Tohnai, N.; Miyata, M., Specific Interaction between Chloroform and the Pockets of Triangular Annulene Derivatives Providing Symmetry Carry-Over Crystallization. Chem. Eur. J. 2009, 15, 13336-13340, S13336/1S13336/22.

53. Hisaki, I.; Sakamoto, Y.; Shigemitsu, H.; Tohnai, N.; Miyata, M.; Seki, S.; Saeki, A.; Tagawa, S., Superstructure-dependent optical and electrical properties of an unusual face-toface, $\pi$-stacked, one-dimensional assembly of dehydrobenzo[12]annulene in the crystalline state. Chem. Eur. J. 2008, 14, 4178-4187. 

TOC:

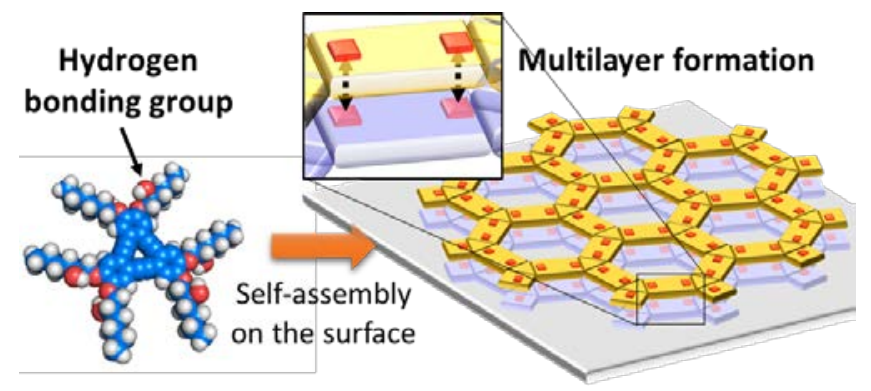

